\title{
RAFT Synthesis of ABA Triblock Copolymers as Ionic Liquid- Containing Electroactive Membranes
}

Tianyu Wu, ${ }^{\dagger}$ Dong Wang, ${ }^{\ddagger}$ Mingqiang Zhang, ${ }^{\dagger}$ James R. Heflin, $^{\dagger}$ Robert B. Moore, ${ }^{\dagger}$ and Timothy E. Long ${ }^{*} \dagger^{+}$

${ }^{\dagger}$ Department of Chemistry, Macromolecules and Interfaces Institute and ${ }^{\ddagger}$ Department of Physics, Virginia Tech, Blacksburg, Virginia 24061, United States

Supporting Information

ABSTRACT: 2-(Dimethylamino)ethyl acrylate (DMAEA) imparts versatile functionality to poly[Sty- $b$ - $(n \mathrm{BA}-c o-$ DMAEA)-b-Sty] ABA triblock copolymers. A controlled synthetic strategy minimized chain transfer reactions and enabled the preparation of high-molecular-weight $\mathrm{ABA}$ triblock copolymers with relatively narrow PDIs between 1.39 and 1.44 using reversible addition-fragmentation chain transfer (RAFT) polymerization. The presence of tertiary amine functionality and their zwitterionic derivatives in the central blocks of the triblock copolymers afforded tunable polarity
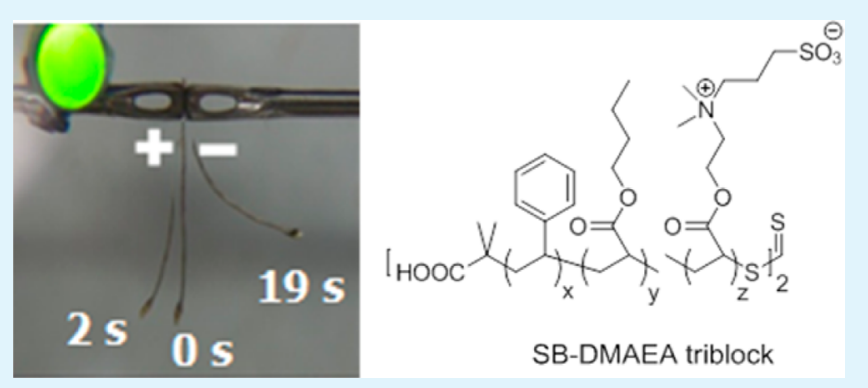
toward ionic liquids. Gravimetric measurements determined the swelling capacity of the triblock copolymers for ionic liquids (IL) 1-ethyl-3-methylimidazolium trifluoromethanesulfonate (EMIm TfO) and 1-ethyl-3-methylimidazolium ethylsulfate (EMIm ES). A correlation of differential scanning calorimetry (DSC), dynamic mechanical analysis (DMA), and small-angle X-ray scattering (SAXS) results revealed the impact of ionic liquid incorporation on the thermal transitions, thermomechanical properties, and morphologies of the triblock copolymers. ILcontaining membranes of DMAEA-derived triblock copolymers and EMIm TfO exhibited desirable rubbery plateau moduli of $\sim 100 \mathrm{MPa}$ and electromechanical actuation to a $4 \mathrm{~V}$ electrical stimulus. Maintaining the mechanical ductility of polymer matrices while increasing their ion-conductivity is paramount for future electroactive devices.

KEYWORDS: acrylate, block copolymer, membrane, ionic liquid, actuator, SAXS

\section{INTRODUCTION}

Electromechanical transducers are devices that exhibit coupling between electrical stimuli and mechanical response. ${ }^{1}$ Over the past decade, electroactive polymers have attracted increasing interest because of their flexibility, lightweight, facile processability, and low fabrication cost. ${ }^{1-4}$ One emerging class of electroactive polymers for electromechanical transducers is ioncontaining polymers such as Nafion. A typical ionic polymer transducer (IPT) assembly consists of an ionic polymer membrane between two conductive electrodes. Akle and Leo proposed that mobile ions within the device accumulated at the electrodes upon charging the IPT, resulting in volume change and bending actuation. ${ }^{5}$ However, typical Nafion-based IPTs operate under hydrated conditions, where the hydrated protons serve as the mobile cations. As a result, their performance deteriorates as water evaporates and electrolysis occurs over time. $^{6}$

Ionic liquids are organic salts that exhibit low melting points, typically below $100{ }^{\circ} \mathrm{C} .{ }^{7}$ Their structural tunability, chemical and thermal stabilities, low vapor pressure, and high ionic conductivity render them promising substitutes for water in IPT applications. ${ }^{8,9}$ Watanabe and co-workers explored the compatibility of various ionic liquid/homopolymer pairs, ${ }^{10}$ and they reported that polymerization of methacrylic monomers in the presence of various ionic liquids resulted in homogeneous ionic gels with desirable high ionic conductivity. ${ }^{11,12}$ Lodge and co-workers also proposed that incorporating ionic liquids into block copolymer matrices afforded mechanical integrity and persistent nanostructure to the ionic liquids. ${ }^{13}$ They further demonstrated the impact of 1-ethyl-3-methylimidazolium trifluoromethanesulfonimide (EMIm-TFSI), an ionic liquid, on the phase behavior and the ionic conductivity of poly(styrene-ethylene oxide) diblock copolymers. ${ }^{14,15}$ Our research group has explored the use of polar sulfobetaine functionalities for desirable interactions with 1-ethyl-3-methylimidazolium ethyl sulfate, EMIm-ES. ${ }^{16}$ We found that the ionic liquid preferentially swelled the zwitterionic domains and significantly impacted thermomechanical performance and ionic conductivity of the zwitterionomer membranes.

More recently, Choi and Colby et al. studied ionic conduction and dielectric response of poly(imidazolium acrylate) homopolymers with various counteranions and alkyl tail lengths on the pendant imidazolium structures. ${ }^{17}$ They employed a physical model of electrode polarization to separate

Received: August 14, 2012

Accepted: November 19, 2012

Published: November 19, 2012 
the ionic conductivity of the poly(imidazolium acrylate)s into number density of conducting ions and their mobility. Their analyses of the static dielectric constants revealed the presence of more ionic aggregates in poly(imidazolium acrylate)s with longer alkyl tails, in agreement with the X-ray scattering results. Gwee, Winey, and Elabd et al. investigated the effect of morphology on the ion transport of this ionic liquid in poly(styrene- $b$-methyl methacrylate). ${ }^{18}$ They observed higher through-plane conductivities in cylindrical morphologies with nonconducting polystyrene cylinders than in lamellar morphologies. They also reported that the ionic conductivity was direction-independent when the morphologies have a continuous conductive path. Imaizumi et al. also further studied the morphology and the viscoelastic properties of ion-gels that consisted of a well-defined poly(styrene- $b$-methyl methacrylate$b$-styrene) triblock copolymer and EMIm-TFSI using atomic force microscopy (AFM) and rheology, respectively. ${ }^{19}$ They demonstrated that the ion-gel actuator bent softly toward the anode under less than $3.0 \mathrm{~V}$ of low applied voltage. However, due to the semicrystalline behavior of poly(ethylene oxide) and the high glass transition temperatures of polystyrene and poly(methyl methacrylate), earlier block copolymers were brittle in the absence of diluents, which complicated device fabrication.

In this study, we synthesized poly(styrene- $b$-acrylate- $b$ styrene) ABA triblock copolymers using the RAFT strategy. The central acrylic block contained quaternizable tertiary amine functionalities and exhibited low glass transition temperatures, which afforded flexible triblock copolymer membranes. The tertiary-amine containing acrylic phase and their zwitterionic derivatives exhibited tunable affinity for ionic liquids. Electromechanical transducers fabricated from these triblock copolymers showed electro-responsiveness. A correlation of differential scanning calorimetry (DSC), dynamic mechanical analysis (DMA), and small-angle X-ray scattering (SAXS) results elucidated the influence of ionic liquid incorporation on the thermal, thermomechanical, and morphological characteristics of the triblock copolymers and their IL-swollen membranes. To the best of our knowledge, this is the first demonstration of non-PEG-based polymer that exhibited both a low glass transition temperature and compatibility with ionic liquids.

\section{EXPERIMENTAL SECTION}

Materials. Carbon disulfide (Aldrich, $\geq 99 \%$ ), tetrabutylammonium hydrogen sulfate (Aldrich, $\geq 99 \%$ ), 1,3-propanesultone (Aldrich, 99+ $\%)$, Stoddard solvents (EMD), chloroform (Fischer Scientific, Optima), acetone (Fischer Scientific, HPLC grade), methanol (Fischer Scientific, HPLC grade), hydrochloric acid (Fischer Scientific, certified ACS Plus), sodium hydroxide (Spectrum Chemical, ACS Reagent), 1ethyl-3-methylimidazolium trifluoromethane-sulfonate (EMIm TfO, IoLiTec Inc., 99\%), and 1-ethyl-3-methylimidazolium ethylsulfate (EMIm ES, Fluka, BASF quality $\geq 95 \%$ ) were used as received. Styrene (Sty, Aldrich, ReagentPlus, $\geq 99 \%$ ), $n$-butyl acrylate ( $n$ BA, Alfa Aesar, $98+\%$ ), and 2-(dimethylamino)ethyl acrylate (DMAEA, Aldrich, 98\%) were deinhibited using neutral alumina columns and further distilled under reduced pressure from calcium hydride. Azobisisobutyronitrile (AIBN, Aldrich, 98\%) was recrystallized from methanol.

RAFT Synthesis of Polystyrene Precursor with S, $S^{\prime}-b i s\left(\alpha, \alpha^{\prime}-\right.$ dimethyl- $\alpha^{\prime \prime}$-acetic acid)-Trithiocarbonate (TTC). The RAFT agent (TTC) was synthesized according to a literature procedure. ${ }^{20}$ In a typical polymerization reaction, styrene $(20 \mathrm{~g}, 0.19 \mathrm{~mol})$, TTC $(16.3 \mathrm{mg}, 58 \mu \mathrm{mol})$, and AIBN $(0.98 \mathrm{mg}, 6.0 \mu \mathrm{mol})$ were charged to a $100 \mathrm{~mL}$, round-bottomed flask equipped with a magnetic stir bar. The reaction mixture was subjected to three freeze-pump-thaw cycles to remove oxygen prior to polymerization and then placed into an oil bath at $65^{\circ} \mathrm{C}$. The reaction product had a targeted molecular weight of $345 \mathrm{~kg} / \mathrm{mol}$ at full conversion. Aliquots were removed from the reaction flask under ultra-high-purity nitrogen atmosphere using a syringe at predetermined time intervals throughout the polymerization. ${ }^{1} \mathrm{H}$ NMR spectroscopy and size exclusion chromatography (SEC) results determined the reaction kinetics and the product molecular weights, respectively. The reaction reached desired monomer conversion after $80 \mathrm{~h}$. The reaction product was precipitated from THF into methanol and dried in a vacuum oven at $65{ }^{\circ} \mathrm{C}$ overnight.

Synthesis of Poly[Sty- $b$-(nBA-co-DMAEA)-b-Sty]. In a typical central block insertion reaction, poly $($ Sty $)$ precursor $(1.0 \mathrm{~g}, 60 \mathrm{~kg} / \mathrm{mol}$, $17 \mu \mathrm{mol}), n$ BA monomer $(6.7 \mathrm{~g}, 52 \mathrm{mmol})$, and AIBN (0.3 mg, 1.8 $\mu \mathrm{mol}$ ) were charged to a $50-\mathrm{mL}$, round-bottomed flask equipped with a magnetic stir bar. The molarity of AIBN was typically $1 / 10$ of the poly(Sty) precursor, while the compositions of the acrylate monomers were either $100 \% n \mathrm{BA}$ or a $90 / 10$ (molar ratio) $n \mathrm{BA} / \mathrm{DMAEA}$ mixture. The reaction mixture was subjected to three freeze-pumpthaw cycles and then placed into an oil bath at $65{ }^{\circ} \mathrm{C}$ for $4 \mathrm{~h}$. The reaction product was finally precipitated from THF into methanol and dried in a vacuum oven at $65{ }^{\circ} \mathrm{C}$ overnight. The triblock copolymer containing 10 mol \% DMAEA in the central block is abbreviated as poly[Sty- $b$-(nBA-co-DMAEA)- $b$-Sty] or as DMAEA triblock copolymer.

Functionalization of Poly[Sty- $b$-(nBA-co-DMAEA)- $b$-Sty] for Sulfobetaine-Containing Derivatives. The molar concentration of DMAEA in poly[Sty- $b$-(nBA-co-DMAEA)- $b$-Sty] was calculated from the ${ }^{1} \mathrm{H}$ NMR spectra as obtained in $\mathrm{CDCl}_{3}$ (see the Supporting Information). In a typical functionalization reaction, poly[Sty- $b-(n \mathrm{BA}-$ co-DMAEA)- $b$-Sty] $(1.0 \mathrm{~g})$ and chloroform $(6 \mathrm{~mL})$ were charged to a $50-\mathrm{mL}$, round-bottomed flask equipped with a magnetic stir bar. Upon dissolution of the neutral triblock copolymer in chloroform, 2 equiv. of 1,3-propanesultone were charged to the reaction mixture. The reaction was refluxed for $20 \mathrm{~h}$, and the final product was precipitated into methanol and vacuum-dried. The sulfobetaine-containing triblock copolymer is abbreviated as poly[Sty- $b$ - $(n \mathrm{BA}-c o-\mathrm{SB}-\mathrm{DMAEA})-b$-Sty] or SB-DMAEA triblock copolymer.

Preparation of Ionic Liquid Swollen Poly(Sty-b-acrylate- $b$ Sty) Membranes. The poly(Sty- $b$-acrylate- $b$-Sty) triblock copolymers had varying compositions in the central blocks, i.e. 100\% $n \mathrm{BA}, 90 / 10$ $n \mathrm{BA} / \mathrm{DMAEA}$, and 90/10 $n \mathrm{BA} / \mathrm{SB}-\mathrm{DMAEA}$. The triblock copolymer membranes were first cast from $0.1 \mathrm{~g} \mathrm{~mL}^{-1}$ chloroform solutions into PTFE molds to achieve film thicknesses of approximately $0.30 \mathrm{~mm}$. The cast films were dried at room temperature overnight and annealed at $120^{\circ} \mathrm{C}$ for $8 \mathrm{~h}$ under vacuum. The dry membranes were immersed into either EMIm TfO or EMIm ES at $60^{\circ} \mathrm{C}$ and stored under vacuum to minimize the impact of moisture uptake. The swollen membranes were periodically removed from the ionic liquid, blotted to remove excess of ionic liquid at the surfaces, and weighed to determine the amount of ionic liquid uptake in the membranes. The mass uptake of the ionic liquid was calculated using the following equation

$$
\text { wt } \% \text { uptake }=\frac{m-m_{0}}{m_{0}} \times 100 \%
$$

where $m_{0}$ is the initial dry mass of the film, and $m$ is the mass of the swollen film at a given time. Once the amount of the ionic liquid uptake in the membranes plateaued, the swollen membranes were transferred to PTFE sheet covered Petri dishes and stored at $25{ }^{\circ} \mathrm{C}$ under vacuum for further characterization.

Instrumentation. ${ }^{1} \mathrm{H}$ NMR spectra were obtained on a Varian Unity $400 \mathrm{MHz}$ spectrometer in $\mathrm{CDCl}_{3}$. Size exclusion chromatography (SEC) determined the molecular weights of the polymers at 35 ${ }^{\circ} \mathrm{C}$ in THF at $1 \mathrm{~mL} \cdot \mathrm{min}^{-1}$ flow rate. The THF SEC system was equipped with a Waters 717plus autosampler, a Waters 515 HPLC pump, three Polymer Laboratories PLGel $5 \mu \mathrm{m}$ Mixed-C columns, and a Waters 2414 differential refractive index detector Relative molecular weights to polystyrene standards for all samples were reported using the Empower Pro software package. 
Scheme 1. Synthesis of Poly[Sty-b-(nBA-co-DMAEA)-b-Sty] Triblock Copolymers, or DMAEA Triblock
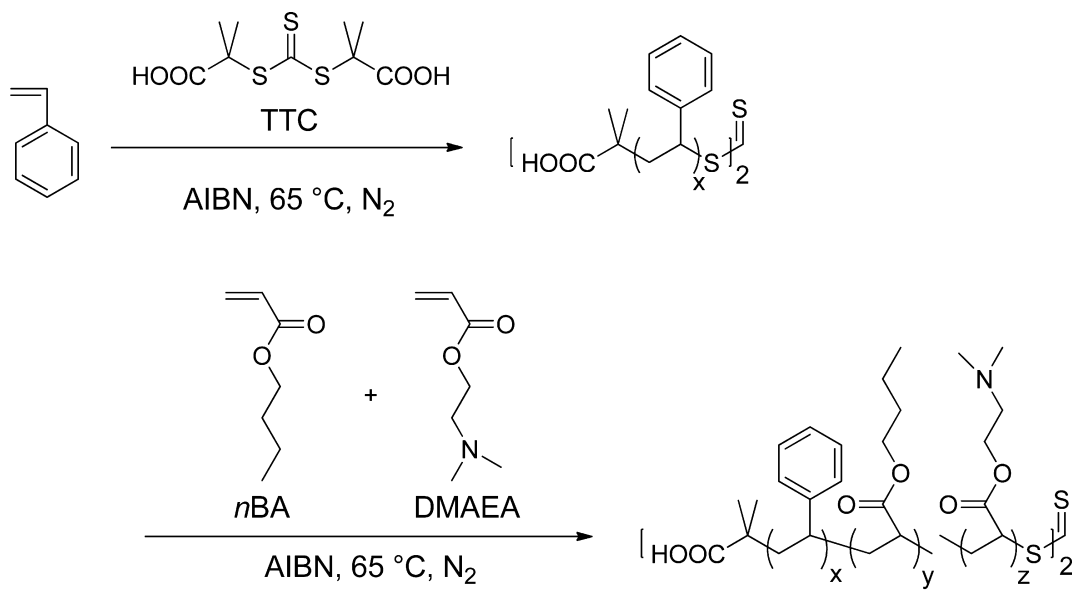

DMAEA triblock

Differential scanning calorimetry (DSC) measurements were performed on a TA Q100 instrument at a heating rate of $10{ }^{\circ} \mathrm{C} /$ $\min$ from $-120^{\circ} \mathrm{C}$. The first heat scans were concluded at $150{ }^{\circ} \mathrm{C}$, and traces of the second heats are reported. Dynamic mechanical analysis (DMA) was performed on a TA Q800 analyzer in tension mode at 1 $\mathrm{Hz}$ frequency, $15 \mu \mathrm{m}$ amplitude, and $3{ }^{\circ} \mathrm{C} / \mathrm{min}$ heating rate from -90 ${ }^{\circ} \mathrm{C}$. Small angle X-ray scattering (SAXS) data were collected using a Rigaku S-Max 3000 SAXS system, equipped with 3 pinholes and a copper rotating anode emitting X-ray with a wavelength of $0.154 \mathrm{~nm}$ $(\mathrm{Cu} \mathrm{K} \alpha)$. Two-dimensional SAXS patterns were obtained using a fully integrated 2D multiwire, proportional counting, gas-filled detector, with an exposure time of $1 \mathrm{~h}$. All SAXS data were analyzed using the SAXSGUI software package to obtain radially integrated SAXS intensity vs scattering vector $q$ plots. For all SAXS experiments, the sample-to-detector distance was $1603 \mathrm{~mm}$, and the $q$ range was calibrated using a silver behenate standard.

Fabrication and Testing of Poly(Sty- $b$-acrylate- $b$-Sty)-Based Electromechanical Transducers. Electromechanical transducers were fabricated in a modified three-step process as previously reported. ${ }^{21,22}$ Specifically, the triblock copolymers were first cast from chloroform solutions at $0.1 \mathrm{~g} \mathrm{~mL}^{-1}$ concentration onto silicone coated Mylar substrates with a drawdown blade set to $500 \mu \mathrm{m}$ gap to achieve $\sim 30 \mu \mathrm{m}$ film thickness. Conductive network composite (CNC) coatings were then deposited onto the polymer membranes ( $\sim 30 \mu \mathrm{m}$ in thickness) using the layer-by-layer (LbL) technique ${ }^{23}$ in order to provide a highly porous electronically and ionically conducting volume for storage of mobile ions. The 30-bilayer LbL CNCs were formed by alternately immersing the membrane in a cationic poly(allylamine hydrochloride) (PAH) aqueous solution (10 $\mathrm{mM}, \mathrm{pH} 4$, Aldrich) and an anionic gold nanoparticle suspension (20 ppm, $\mathrm{pH}$ 9, diameter $=3 \mathrm{~nm}$, zeta potential $=-40 \mathrm{mV}$, Purest Colloids Inc.) The alternate cationic and anionic layers were bound tightly through electrostatic self-assembly, generating uniform coatings with well-controlled thickness at the nanoscale through adjusting the number of layers. The membranes were subsequently immersed into EMIm TfO ionic liquid at $60{ }^{\circ} \mathrm{C}$ for $28 \mathrm{~h}$. Gold foil $(\sim 50 \mathrm{~nm}$ in thickness) was finally hot pressed onto the outer surfaces of the devices at $60{ }^{\circ} \mathrm{C}$ and under $700 \mathrm{psi}$ pressure for $20 \mathrm{~s}$ to serve as the electrodes. The actuator was then cut into $1 \mathrm{~mm} \times 8 \mathrm{~mm}$ strips and subjected to a $4 \mathrm{~V} \mathrm{DC}$ step voltage at $23{ }^{\circ} \mathrm{C}$ and $70 \%$ relative humidity. The bending response of the electromechanical transducer was recorded using a Sony HD Camcorder (Model HXR-MC1) with 30 fps.

\section{RESULTS AND DISCUSSIONS}

RAFT Synthesis of Polystyrene and Poly(Sty-bacrylate- $b$-Sty $)$ with $\mathrm{S}, \mathrm{S}^{\prime}$-Bis $\left(\alpha, \alpha^{\prime}\right.$-dimethyl- $\alpha^{\prime \prime}$-acetic acid)-trithiocarbonate (TTC). The past 15 years have witnessed rapid growth in the development and understanding of controlled radical polymerization (CRP) techniques, such as NMP ${ }^{24,25}{ }^{2 T R P},{ }^{26}$ and RAFT. ${ }^{27}$ These strategies have enabled the incorporation of many functional monomers, which are typically incompatible with living ionic polymerization techniques, into polymers of advanced architectures and narrow polydispersity. Lai et al. first reported TTC-mediated RAFT polymerization of a variety of monomers and demonstrated the suitability of the short chain precursors for central block insertion reactions. ${ }^{20}$ Ran et al. photoinitiated the synthesis of high molecular weight poly(Sty-b-nBA-b-Sty) triblock copolymers in the presence of TTC and demonstrated good control. ${ }^{28}$ However, to the best of our knowledge, there is not a precedence of incorporating tertiary-amine containing acrylates into the central blocks of high molecular weight poly(Sty- $b$ acrylate- $b$-Sty)-type triblock copolymers for flexible membrane applications.

Scheme 1 illustrates the TTC-mediated RAFT polymerization of poly[Sty-b-(nBA-co-DMAEA)-b-Sty], where the central block contained a mixture of $n \mathrm{BA}$ and DMAEA units. Poly(Sty) precursor was first synthesized in bulk at a AIBN:TTC molar ratio of 1:10. The reaction exhibited pseudo-first-order kinetics, and the molecular weights of the poly(Sty) products increased linearly as a function of the monomer conversion. Details of the reaction kinetics for TTCmediated homopolymerization of styrene are provided in the Supporting Information.

The suitability of the poly(Sty) precursor for central block insertion depended on the choice of the acrylic monomers. Although the synthesis of poly(Sty-b-nBA-b-Sty) was well controlled as indicated in Table S2 in the Supporting Information, central block insertion reactions involving DMAEA presented challenges. ${ }^{29}$ Conventional free radical polymerization of $n \mathrm{BA}$ in the presence of triethylamine (TEA) revealed that the propagating acrylic radicals chain transferred to TEA with a chain transfer constant $\left(C_{\mathrm{s}}\right)$ of 0.0240 , which is on the same order of magnitude as chain transfer from poly (methyl acrylate) radicals to TEA $\left(C_{\mathrm{s}} \approx 0.0400\right) .{ }^{30}$ Details of these calculations are also provided in the Supporting Information.

Since the success of controlled radical polymerization techniques relies on minimizing the propensity of propagating 
chain end to undergo undesirable chain transfer or termination, we targeted $10 \mathrm{~mol} \%$ DMAEA in the acrylate monomer feed and reduced the molar AIBN:TTC ratio to $1: 20$ to improve the control over the central block insertion reaction. Figure 1

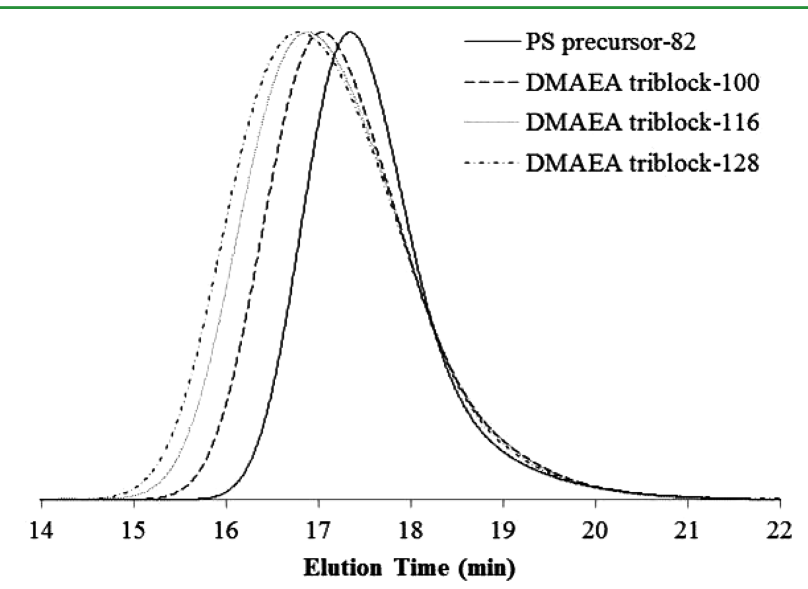

Figure 1. SEC traces for the PS precursor and DMAEA-containing triblock copolymers of varying central block lengths. All central blocks contained 10 mol \% DMAEA. The abbreviations indicate the weightaverage molecular weights of the final products (in $\mathrm{kg} \mathrm{mol}^{-1}$ ).

shows the SEC chromatograms for the poly(Sty) precursor and poly [Sty- $b$-(nBA-co-DMAEA)- $b$-Sty] synthesized under these reaction conditions. The relative molecular weights of these polymers to poly(Sty) standards and their PDIs are summarized in Table $\mathrm{S} 1$ in the Supporting Information. Three triblock copolymers were sampled from the same reaction at $4 \%, 6 \%$, and $12 \%$ monomer conversions. The shift of the SEC traces toward shorter elution time with increasing acrylate conversion indicated the growth of the central blocks for the triblock copolymers. Relatively narrow PDIs between 1.39 and 1.44 for the triblock copolymers confirmed that the radical polymerization of the acrylates behaved in a controlled fashion. The tailing at the low molecular weight end of the distribution for the triblock copolymers suggested that chain transfer from the acrylic radicals to DMAEA was not completely eliminated during the polymerization process. Thus, the triblock copolymers presumably contained some poly[Sty- $b$-(nBA-co-DMAEA)] diblock contaminants. Nevertheless, a viable strategy for minimizing chain transfer to DMAEA resulted in a library of poly[Sty- $b-(n \mathrm{BA}-c o$-DMAEA $)$ $b$-Sty] triblock copolymers for morphological studies and flexible host membranes for ionic liquids.

Functionalization of Poly[Sty- $b$-(nBA-co-DMAEA)- $b$ Sty] for Sulfobetaine-Containing Derivatives. Scheme 2 illustrates the functionalization of poly[Sty- $b-(n \mathrm{BA}-c o-$ DMAEA)- $b$-Sty] with 1,3-propanesultone. The characteristic ${ }^{1} \mathrm{H}$ NMR resonances for both neutral and sulfobetainecontaining triblock copolymers are shown in Figure S4 in the Supporting Information. The ${ }^{1} \mathrm{H}$ NMR spectroscopic results confirmed complete conversion of DMAEA to its zwitterionic derivative in the triblock copolymer.

Swelling of Poly(Sty- $b$-acrylate- $b$-Sty) Triblock Copolymers with Ionic Liquids. The poly(Sty- $b$-acrylate- $b$-Sty) triblock copolymers varied in their central block compositions. Poly (Sty- $b-n \mathrm{BA}-b$-Sty) contained a pure $n \mathrm{BA}$ central block, and both the DMAEA triblock and the SB-DMAEA triblock contained $90 \mathrm{~mol} \% n \mathrm{BA}$ in their central blocks. The SBDMAEA triblock was directly derived from the DMAEA triblock. All three triblock copolymers had comparable relative molecular weights to polystyrene standards, and the values are listed in Table S2 in the Supporting Information. Figure 2 illustrates the chemical structures of the ionic liquids, and both EMIm TfO and EMIm ES contained 1-ethyl-3-methylimidazolium cations.

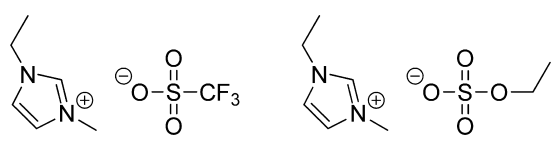
(a) EMIm TfO
(b) EMIm ES

Figure 2. Chemical structures of the ionic liquids: (a) EMIm TfO and (b) EMIm ES.

IL swelling studies were performed in a vacuum oven to reduce the impact of moisture uptake, and a $60{ }^{\circ} \mathrm{C}$ swelling temperature accelerated IL uptake. Figures 3 and 4 show the

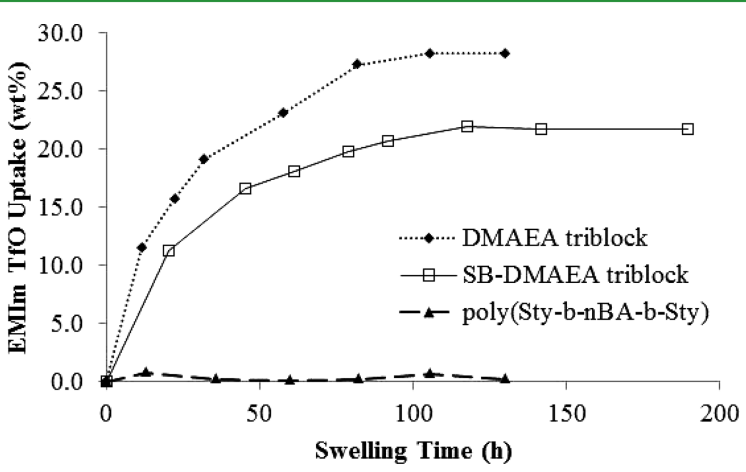

Figure 3. Swelling profiles for the triblock copolymers with EMIm TfO at $60{ }^{\circ} \mathrm{C}$.

Scheme 2. Functionalization of DMAEA Triblock Copolymer with 1,3-Propanesultone to Form Poly[Sty- $b$ - $(n B A-c o-$ SBDMAEA)- $b$-Sty], or SB-DMAEA Triblock

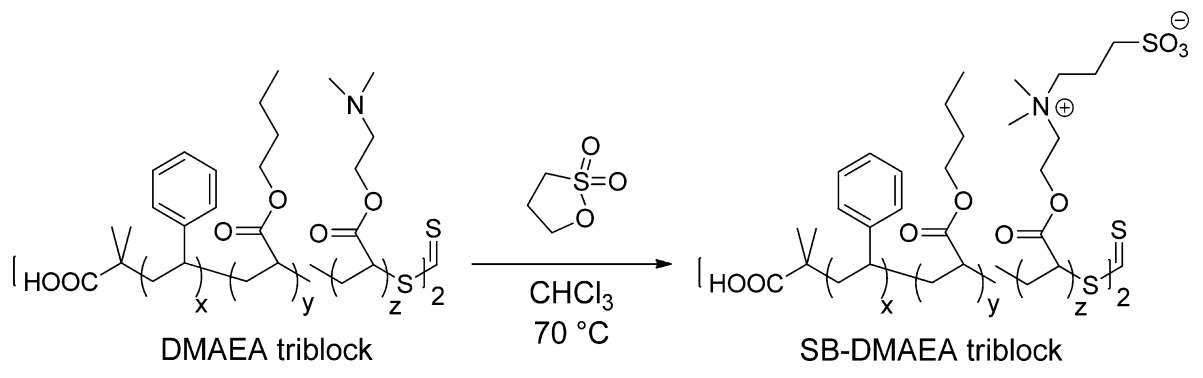




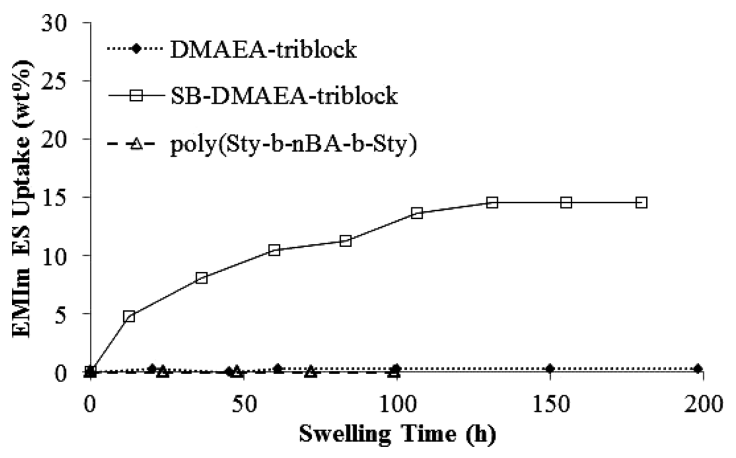

Figure 4. Swelling profiles for the triblock copolymers with EMIm ES at $60{ }^{\circ} \mathrm{C}$.

swelling profiles for the triblock copolymers in EMIm TfO and EMIm ES, respectively. Poly(Sty- $b-n$ BA- $b$-Sty) did not absorb appreciable ionic liquid ( $\sim 0 \mathrm{wt} \%)$ in either EMIm TfO or EMIm ES over $100 \mathrm{~h}$. Upon $10 \mathrm{~mol} \%$ DMAEA incorporation into the central block, the tertiary amine-containing triblock copolymer exhibited significantly improved affinity toward EMIm TfO with a 28 wt \% uptake at equilibrium. However, it did not absorb EMIm ES; 0 wt \% IL uptake was observed over $200 \mathrm{~h}$.

In comparison, the SB-DMAEA triblock copolymer showed affinity toward both EMIm TfO and EMIm ES. While the zwitterionic triblock copolymer absorbed less EMIm TfO $(\sim 22$ wt \%) at a slower rate than the DMAEA triblock copolymer, swelling with EMIm ES achieved an equilibrium uptake of $\sim 15$ wt \%. Tigelaar et al. studied water and ionic liquid uptake behavior of aromatic rigid-rod elastomeric polymers with imidazolium-trifluoromethanesulfonimide (imid-TFSI), imidazolium-trifluoromethanesulfonic acid (imid- $\mathrm{CF}_{3} \mathrm{SO}_{3} \mathrm{H}$ ) and imidazolium-sulfuric acid (imid- $\mathrm{H}_{2} \mathrm{SO}_{4}$ ). They reported similar dependence of equilibrium ionic liquid uptake on the pairing between the polymer matrix and the ionic liquid, ${ }^{31}$ and they postulated that the observed difference was due to size differences between the ionic liquids and the free volume between the polymer chains. Nevertheless, our experimental results suggested that it is possible to adjust the affinity of the triblock copolymers toward polar guest molecules such as the ionic liquids.

Thermal and Thermomechanical Properties of Ionic Liquid Swollen Poly(Sty-b-acrylate- $b$-Sty) Membranes. DSC results revealed the influence of the ionic liquids on the thermal transitions of the triblock copolymers. The glass transition temperatures of the triblock copolymers and their composites with ionic liquids, as well as the melting points of both EMIm TfO and EMIm ES, are summarized in Table 1. EMIm TfO exhibited a glass transition temperature of $-95{ }^{\circ} \mathrm{C}$ and a melting temperature of $-13{ }^{\circ} \mathrm{C}$, whereas EMIm ES showed a glass transition temperature of $-82{ }^{\circ} \mathrm{C}$ only, in agreement with earlier literature. ${ }^{32,33}$ The presence of two $T_{\mathrm{g}}$ values for the triblock copolymers indicated microphase separation, which was independent of ionic liquid content. The higher $T_{\mathrm{g}}$ at $\sim 100{ }^{\circ} \mathrm{C}$ corresponded to the poly(Sty) phase, and the lower $T_{\mathrm{g}}$ at approximately $-40{ }^{\circ} \mathrm{C}$ corresponded to the acrylic phase.

The DSC results showed that the presence of either ionic liquid in the triblock copolymers had minimal impact on the glass transition temperatures of the poly(Sty) phase, suggesting that the ionic liquid resided mainly in the acrylic phase. The lower $T_{\mathrm{g}}$ values of the triblock copolymers increased noticeably
Table 1. Thermal Transitions for the Triblock Copolymers with and without Ionic Liquids

\begin{tabular}{cllll}
\multicolumn{1}{c}{ matrix } & \multicolumn{1}{c}{ ionic liquid } & $T_{\mathrm{g}, 1}\left({ }^{\circ} \mathrm{C}\right)$ & $T_{\mathrm{g}, 2}\left({ }^{\circ} \mathrm{C}\right)$ & $\begin{array}{c}T_{\mathrm{m}}{ }^{a} \\
\left({ }^{\circ} \mathrm{C}\right)\end{array}$ \\
$\begin{array}{c}\text { DMAEA } \\
\text { triblock } \\
\text { copolymers }\end{array}$ & $\begin{array}{c}\text { without IL } \\
\text { EMIm TfO (17-28 wt }\end{array}$ & -46 & 103 & $\mathrm{ND}$ \\
$\begin{array}{c}\text { SB-DMAEA } \\
\text { triblock }\end{array}$ & without IL & -36 & $106 \pm 1$ & $\mathrm{ND}$ \\
copolymers & EMIm TfO (22 wt \%) & -30 & 105 & $\mathrm{ND}$ \\
& EMIm ES (15 wt \%) & -34 & 106 & $\mathrm{ND}$ \\
& EMIm TfO & -95 & $\mathrm{ND}$ & $\mathrm{ND}$ \\
& EMIm ES & -83 & $\mathrm{ND}$ & $\mathrm{ND}$
\end{tabular}

${ }^{a} \mathrm{ND}=$ not detected.

upon EMIm TfO incorporation, while SB-DMAEA triblock copolymer showed minimal change with $\sim 15$ wt \% EMIm ES incorporation. Koberle and Laschewsky studied the impact of stoichiometric amounts of $\mathrm{NaI}$ on the glass transition temperatures of sulfobetaine-containing homopolymers. ${ }^{34}$ They observed increases in the glass transition temperatures of the homogeneous blends and attributed those increases to strong attractive interactions between the low molecular weight ions and the polymer-bound betaine functionalities. Similar molecular interactions presumably contribute to an increase in the $T_{\mathrm{g}}$ of SB-DMAEA triblock copolymer upon EMIm TfO incorporation. However, these interactions were not expected in neutral DMAEA triblock copolymers. Because EMIm TfO exhibited a melting point of $-12{ }^{\circ} \mathrm{C}$, higher than the $T_{\mathrm{g}}$ values of the acrylic phase, the addition of EMIm TfO may presumably reduce the amount of free volume in the triblock copolymers at temperatures below the melting point. As a result, the $T_{\mathrm{g}}$ values of the acrylic phase increased. Nevertheless, considering that poly(Sty- $b-n \mathrm{BA}-b-S t y)$ showed $\sim 0$ wt $\%$ uptake of either ionic liquid after $100 \mathrm{~h}$ of swelling, the impact of the chemical modifications in the central blocks of the triblock copolymers on their affinity toward polar guest molecules, such as the ionic liquids, was unexpected.

Figure 5 compares the DMA traces for the triblock copolymers with and without EMIm TfO. Rubbery plateaus

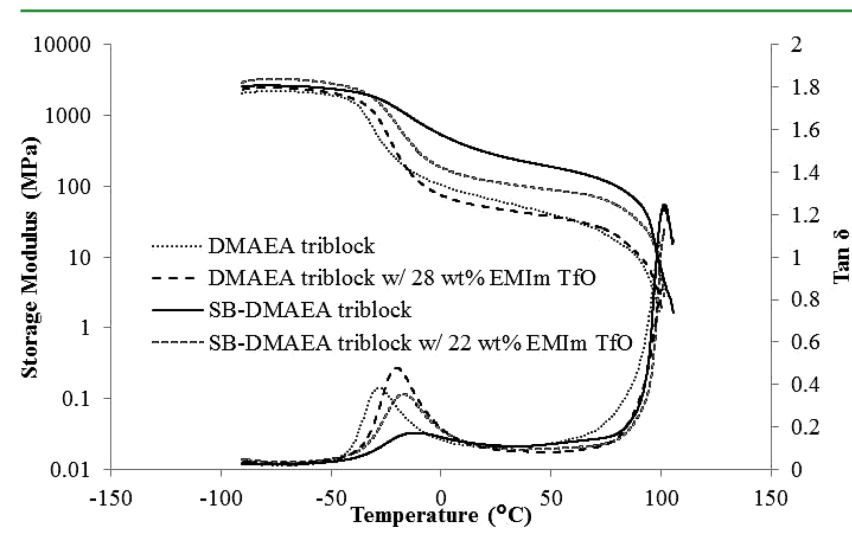

Figure 5. DMA traces for DMAEA triblock and SB-DMAEA triblock copolymers with and without EMIm TfO.

spanning from -20 to $100{ }^{\circ} \mathrm{C}$ were observed for all samples. The presence of the ionic liquid only affected the glass transition temperatures of the acrylic phase, but the flow temperatures, which were associated with the $T_{\mathrm{g}}$ of the poly(Sty) phase, remained unchanged. This is in agreement with DSC data. The SB-DMAEA triblock copolymer in the neat 
state exhibited a plateau modulus about one order of magnitude higher than the neutral analog. However, upon ionic liquid incorporation, the plateau modulus for the sulfobetainecontaining triblock copolymer decreased significantly, while the DMAEA-containing triblock copolymer remained unchanged. We attributed this decrease in the storage modulus for the zwitterionic triblock copolymer to the dissociation of the strong electrostatic interactions between the sulfobetaine functionalities in the presence of ionic liquid. Since these electrostatic interactions are only present in the SB-DMAEA triblock copolymer, the observed different influences of EMIm TfO on the plateau moduli of the triblock copolymers was expected. Both IL composite membranes of DMAEA triblock and SB-DMAEA triblock with EMIm TfO had plateau moduli of $\sim 100 \mathrm{MPa}$, which was desirable for high moduli electroactive membranes.

Morphologies of Neat and Ionic Liquid Swollen Poly(Sty-b-acrylate- $b$-Sty) Membranes. Figure 6 compares

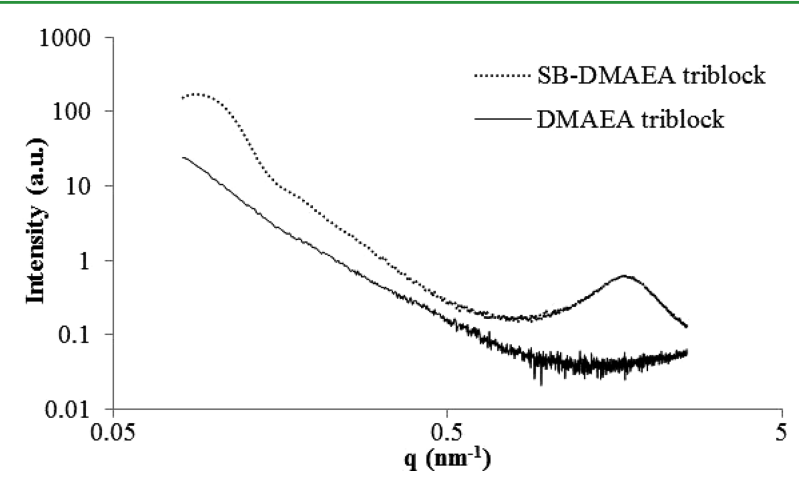

Figure 6. SAXS analysis of neat DMAEA- and SB-DMAEA-containing triblock copolymers in the absence of ionic liquids (ILs).

SAXS traces for both DMAEA triblock and SB-DMAEA triblock copolymers without ionic liquid incorporation. The scattering trace for DMAEA triblock copolymer appeared featureless, whereas a distinctive scattering peak at $\sim 1.5 \mathrm{~nm}^{-1}$ was observed for the SB-DMAEA triblock copolymer. We previously attributed a similar scattering peak for a zwitterionic random copolymer to the difference in electron densities between the zwitterionic aggregates and their surrounding matrix. $^{35}$ In contrast, the presence of the sulfobetaine functionalities in the acrylic central blocks of the SB-DMAEA triblock copolymer likely resulted in ionic aggregation within the acrylic phase. In addition, scattering signals at $\sim 0.09$ and $\sim 0.18 \mathrm{~nm}^{-1}$ for the SB-DMAEA triblock copolymer indicated that the strong electrostatic interactions between the sulfobetaine functionalities enhanced the long-range microphase separation of the triblock copolymer. Transmission electron microscopy (TEM) for the SB-DMAEA triblock copolymer, as shown in Figure S7 in the Supporting Information, agreed with the SAXS conclusions.

Figure 7 compares SAXS profiles for DMAEA triblock copolymer with and without EMIm TfO. The DMAEA triblock copolymer exhibited 0 wt \% EMIm ES uptake over an extended period of time. Both DSC and DMA results suggested that EMIm TfO resided mainly in the acrylic phase of the composite membranes. SAXS analysis for the neat DMAEA triblock copolymer indicated the absence of a well-defined morphology. However, upon EMIm TfO incorporation into the acrylic phase, a scattering peak at $\sim 0.09 \mathrm{~nm}^{-1}$ and a scattering

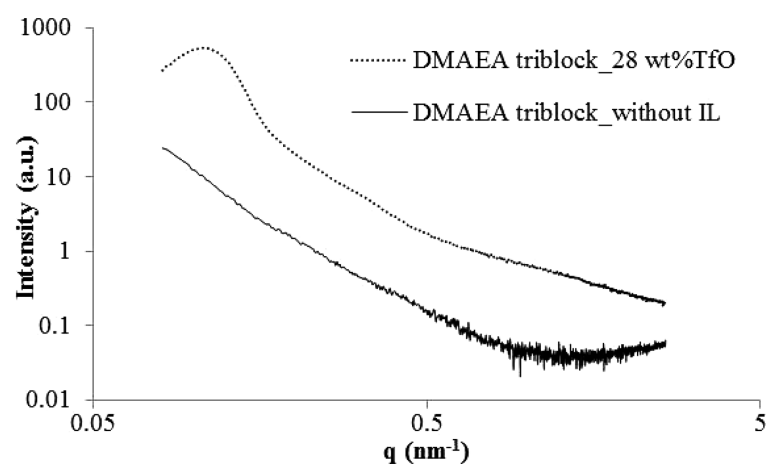

Figure 7. SAXS for DMAEA triblock copolymer without IL and with 28 wt \% EMIm TfO.

shoulder at $\sim 0.18 \mathrm{~nm}^{-1}$ appeared for the composite membrane. The SAXS results suggested that the presence of EMIm TfO enhanced incompatibility between the polystyrene phase and the acrylic phase, which led to more well-defined morphology for the DMAEA triblock copolymer membrane.

Figure 8 shows SAXS traces for SB-DMAEA triblock copolymer and composite membranes containing $22 \mathrm{wt} \%$

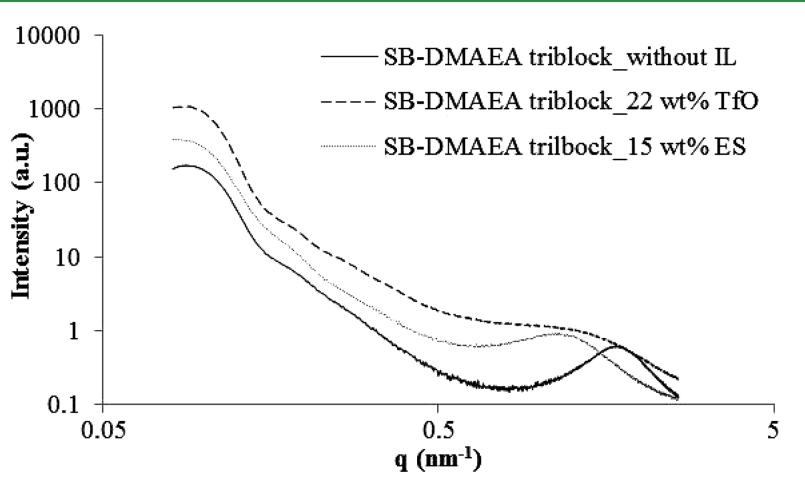

Figure 8. SAXS for SB-DMAEA triblock copolymer films without ionic IL, with 22 wt \% EMIm TfO and with 15 wt \% EMIm ES.

EMIm TfO and 15 wt \% EMIm ES, respectively. The scattering features suggested that the long-range ordered morphologies of the neat SB-DMAEA triblock copolymer persisted upon ionic liquid incorporation. However, the ionomer peak shifted to lower $q$, which corresponded to an increase in the intercluster spacing with 15 wt \% EMIm ES incorporation, and the peak broadened significantly with 22 wt \% EMIm TfO. Gierke et al. attributed a similar increase in the mean intercluster spacing for water-swollen Nafion membranes to the coalescence of adjacent ion clusters. ${ }^{36}$ Bennett et al. studied the impact of EMIm TfO and EMIm TFSI on the morphology of Nafion membranes. ${ }^{37}$ They reported that the hydrophilic EMIm TfO was absorbed into the ionic clusters, whereas the hydrophobic EMIm TFSI resided at least partially in the nonpolar polymer matrix. Here, we attributed the difference in the SAXS traces for the EMIm TfO- and EMIm ES-swollen SB-DMAEA triblock copolymer membranes to the difference in polarity between the two ionic liquids. The tunability of the tertiary amine functionalities in the DMAEA triblock copolymer allowed us to adjust the polarity of the triblock copolymer and affinity toward polar guest molecules such as ionic liquids.

Electroresponsiveness of Poly(Sty- $b$-acrylate- $b$-Sty)Based Actuators. DMAEA triblock, SB-DMAEA triblock, and poly(Sty- $b-n$ BA- $b$-Sty) were fabricated into electro- 


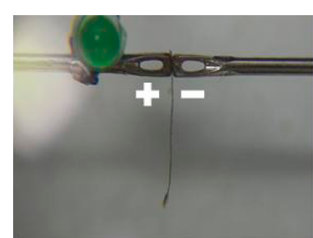

$0 \mathrm{~s}$

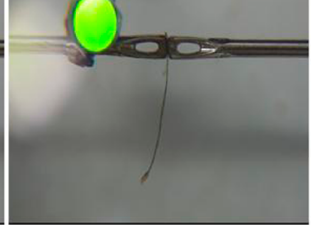

$2 \mathrm{~s}$

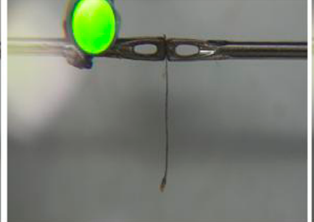

$6 \mathrm{~s}$

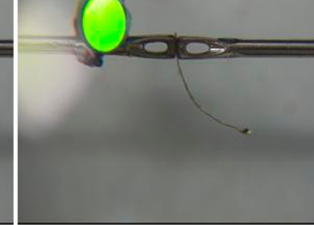

$19 \mathrm{~s}$

Figure 9. Images of electromechanical transducers fabricated from the SBDMAEA triblock copolymer with 58 wt \% EMIm TfO.

mechanical transducers according to a modified literature protocol. $^{22}$ The actuators all contained higher ionic liquid contents than the neat membranes due to the presence of conductive network composite (CNC) coatings on their surfaces. Figure 9 depicts the still images of the actuator fabricated from SB-DMAEA triblock copolymers under a $4 \mathrm{~V}$ step applied potential. Appreciable bending actuation was observed upon application of external electrical stimuli for both EMIm TfO-swollen DMAEA triblock and EMIm TfO-swollen SB-DMAEA triblock, but not for poly(Sty- $b-n$ BA- $b$-Sty), which did not show EMIm TfO uptake. Surprisingly, SB-DMAEA triblock copolymer swollen with EMIm ES also did not exhibit electro-responsiveness. As seen in Figure 9, the actuator initially bent toward the anode and later reversed the motion toward the cathode under the unipolar step potential. This bidirectional actuation behavior agreed with previous observation in ionic liquid-based actuation and was distinct from typical backrelaxation behavior. ${ }^{38}$ It was ascribed to a faster motion of the smaller mobile cations followed by a slower motion of the larger mobile anions.

Because the bending actuation resulted from the accumulation of the mobile ions on both sides of the actuator, the bending curvature should be proportional to the charging of an equivalent resistor-capacitor (RC) circuit. Liu et al. simulated the ion motion in a similarly fabricated Nafion-based IPT using a two-branch parallel RC circuit and obtained the characteristic time constants for both the cation motion and the anion motion. ${ }^{38}$ They also successfully derived the maximum actuation curvature for their device. Figure 10 shows that the equivalent RC circuit fits the actuation curvature for our

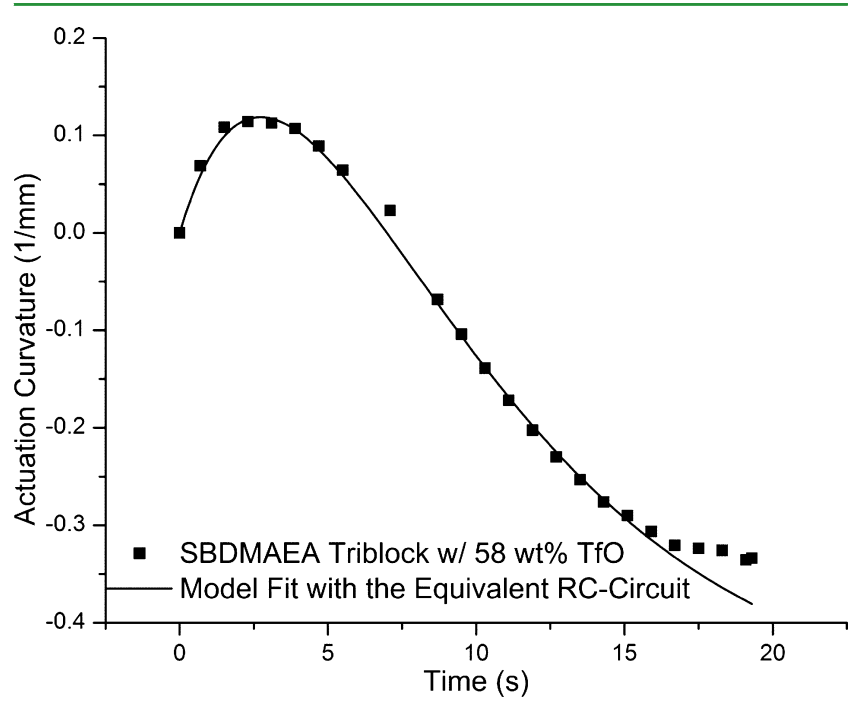

Figure 10. Actuation curvature for electromechanical transducer fabricated from the SBDMAEA triblock copolymer with 58 wt \% EMIm TfO and the model fit with an equivalent RC circuit $^{38}$.
SBDMAEA triblock copolymer-based actuator very well. Detailed fitting procedure and the fitting parameters are provided in the Supporting Information. Although a more comprehensive evaluation of the actuation curvature and the bending force is the subject of a future study, the current results indicated that the incorporation of DMAEA into the central blocks of poly(Sty-b-acrylate- $b$-Sty) enhanced affinity toward ionic liquids and enabled the electroresponsiveness of the triblock copolymer/EMIm TfO composite membranes.

\section{CONCLUSIONS}

We demonstrated the synthesis of high-molecular-weight poly $[$ Sty- $b$-( $n \mathrm{BA}$-co-DMAEA)- $b$-Sty] with relatively narrow PDIs between 1.39 and 1.44 employing the RAFT polymerization strategy. The presence of the tertiary amine functionality in the central block of the triblock copolymers afforded tunable polarity toward polar guest molecules, such as ionic liquids. Both DMAEA triblock and SB-DMAEA triblock copolymers showed much improved affinity toward EMIm $\mathrm{TfO}$, while their $n \mathrm{BA}$ counterpart did not absorb ionic liquid. Thermal and thermomechanical analyses of the block copolymer and their composite membranes revealed that they all had two distinctive glass transition temperatures at $\sim-40$ ${ }^{\circ} \mathrm{C}$ and $\sim 100{ }^{\circ} \mathrm{C}$. The lower $T_{\mathrm{g}}$ corresponded to the acrylate phase, where the ionic liquid resided, while the higher $T_{\mathrm{g}}$ corresponded to the IL-free poly(Sty) phase, which served as the physical cross-links in the membranes. The triblock copolymer/EMIm TfO membranes exhibited rubbery plateaus between -20 and $100{ }^{\circ} \mathrm{C}$ and plateau moduli of $\sim 100 \mathrm{MPa}$, both of which are desirable for applications in electromechanical transducers. SAXS results revealed that both quaternization of DMAEA to SB-DMAEA functionalities and the incorporation of ionic liquids into the acrylic phase facilitated the development of long-range ordered morphology in the triblock copolymers. Actuators fabricated from poly[Sty$b$-(nBA-co-SBDMAEA)-b-Sty] indeed showed electro-responsiveness. Despite the high chain transfer constants for acrylic radicals to tertiary amines, the RAFT synthesis of poly[Sty- $b$ (nBA-co-DMAEA)-b-Sty] provided tunability for further understanding the structure-morphology-performance relationship of polymer-based electromechanical transducers.

\section{ASSOCIATED CONTENT}

\section{Supporting Information}

reaction kinetics for the RAFT synthesis of polystyrene precursors; the structural confirmation of the triblock copolymers, and their molecular weight data; DSC traces for the triblock copolymer/ionic liquid binary compositions. This material is available free of charge via the Internet at http:// pubs.acs.org. 


\section{AUTHOR INFORMATION}

\section{Corresponding Author}

*E-mail telong@vt.edu.

Notes

The authors declare no competing financial interest.

\section{ACKNOWLEDGMENTS}

This material is based on work supported by the U.S. Army Research Office under Grant W911NF-07-1-0452 Ionic Liquids in Electro-Active Devices (ILEAD) MURI.

\section{REFERENCES}

(1) Duncan, A. J.; Leo, D. J.; Long, T. E. Macromolecules 2008, 41 (21), 7765-7775.

(2) Pelrine, R.; Kornbluh, R.; Pei, Q. B.; Joseph, J. Science 2000, 287 (5454), 836-839.

(3) Shankar, R.; Krishnan, A. K.; Ghosh, T. K.; Spontak, R. J. Macromolecules 2008, 41 (16), 6100-6109.

(4) Gao, R.; Wang, D.; Heflin, J. R.; Long, T. E. J. Mater. Chem. 2012, 22 (27), 13473-13476.

(5) Akle, B. J.; Leo, D. J. Smart Materials and Structures 2007, 16 (4), 1348.

(6) Bennett, M. D.; Leo, D. J. Sens. Actuators, A 2004, 115 (1), 7990.

(7) Visser, A. E.; Swatloski, R. P.; Reichert, W. M.; Mayton, R.; Sheff, S.; Wierzbicki, A.; Davis, J. H.; Rogers, R. D. Environ. Sci. Technol. 2002, 36 (11), 2523-2529.

(8) Green, M. D.; Long, T. E. Polym. Rev. 2009, 49 (4), 291-314.

(9) Armand, M.; Endres, F.; MacFarlane, D. R.; Ohno, H.; Scrosati, B. Nat. Mater. 2009, 8 (8), 621-629.

(10) Ueki, T.; Watanabe, M. Macromolecules 2008, 41 (11), 37393749.

(11) Noda, A.; Watanabe, M. Electrochim. Acta 2000, 45 (8-9), $1265-1270$

(12) Susan, M. A. B. H.; Kaneko, T.; Noda, A.; Watanabe, M. J. Am. Chem. Soc. 2005, 127 (13), 4976-4983.

(13) Lodge, T. P. Science 2008, 321 (5885), 50-51.

(14) Meli, L.; Lodge, T. P. Macromolecules 2009, 42 (3), 580-583.

(15) Simone, P. M.; Lodge, T. P. ACS Appl. Mater. Interfaces 2009, 1

(12), 2812-2820.

(16) Brown, R. H.; Duncan, A. J.; Choi, J. H.; Park, J. K.; Wu, T. Y.; Leo, D. J.; Winey, K. I.; Moore, R. B.; Long, T. E. Macromolecules 2010, 43 (2), 790-796.

(17) Choi, U. H.; Lee, M.; Wang, S.; Liu, W.; Winey, K. I.; Gibson, H. W.; Colby, R. H. Macromolecules 2012, 45 (9), 3974-3985.

(18) Gwee, L.; Choi, J.-H.; Winey, K. I.; Elabd, Y. A. Polymer 2010, 51 (23), 5516-5524.

(19) Imaizumi, S.; Kokubo, H.; Watanabe, M. Macromolecules 2011, 45 (1), 401-409.

(20) Lai, J. T.; Filla, D.; Shea, R. Macromolecules 2002, 35 (18), 6754-6756.

(21) Liu, S.; Montazami, R.; Liu, Y.; Jain, V.; Lin, M.; Heflin, J. R.; Zhang, Q. M. Appl. Phys. Lett. 2009, 95 (2), 023505/1-023505/3.

(22) Liu, S.; Montazami, R.; Liu, Y.; Jain, V.; Lin, M.; Zhou, X.; Heflin, J. R.; Zhang, Q. M. Sens. Actuators, A 2010, A157 (2), 267275.

(23) Decher, G.; Hong, J. D. Makromol. Chem., Macromol. Symp. 1991, 46, 321-327.

(24) Hawker, C. J.; Bosman, A. W.; Harth, E. Chem. Rev. 2001, 101

(12), 3661-3688.

(25) Mather, B. D.; Baker, M. B.; Beyer, F. L.; Berg, M. A. G.; Green, M. D.; Long, T. E. Macromolecules 2007, 40 (19), 6834-6845.

(26) Matyjaszewski, K.; Xia, J. Chem. Rev. 2001, 101 (9), 2921-2990.

(27) Chiefari, J.; Chong, Y. K.; Ercole, F.; Krstina, J.; Jeffery, J.; Le, T. P. T.; Mayadunne, R. T. A.; Meijs, G. F.; Moad, C. L.; Moad, G.; Rizzardo, E.; Thang, S. H. Macromolecules 1998, 31 (16), 5559-5562.
(28) Ran, R.; Yu, Y.; Wan, T. J. Appl. Polym. Sci. 2007, 105 (2), 398404.

(29) Truong, N. P.; Jia, Z.; Burges, M.; McMillan, N. A. J.; Monteiro, M. J. Biomacromolecules 2011, 12 (5), 1876-1882.

(30) Ueda, A.; Nagai, S. In Polymer Handbook, fourth ed.; Brandrup, J., Immergut, E. H., Grulke, E. A., Eds.; John Wiley \& Sons: New York, 1999; p II/113.

(31) Tigelaar, D. A.; Waldecker, J. R.; Peplowski, K. M.; Kinder, J. D. Polymer 2006, 47 (12), 4269-4275.

(32) Bonhôte, P.; Dias, A.-P.; Papageorgiou, N.; Kalyanasundaram, K.; Grätzel, M. Inorg. Chem. 1996, 35 (5), 1168-1178.

(33) Vila, J.; Franjo, C.; Pico, J. M.; Varela, L. M.; Cabeza, O. Port. Electrochim. Acta 2007, 25 (1), 163-172.

(34) Koberle, P.; Laschewsky, A. Macromolecules 1994, 27 (8), 2165-2173.

(35) Wu, T.; Beyer, F. L.; Brown, R. H.; Moore, R. B.; Long, T. E. Macromolecules 2011, 44 (20), 8056-8063.

(36) Gierke, T. D.; Munn, G. E.; Wilson, F. C. Perfluorinated Ionomer Membranes; American Chemical Society: Washington, D.C., 1982; Vol. 180, pp 195-216.

(37) Bennett, M. D.; Leo, D. J.; Wilkes, G. L.; Beyer, F. L.; Pechar, T. W. Polymer 2006, 47 (19), 6782-6796.

(38) Liu, Y.; Liu, S.; Lin, J.; Wang, D.; Jain, V.; Montazami, R.; Heflin, J. R.; Li, J.; Madsen, L.; Zhang, Q. M. Appl. Phys. Lett. 2010, 96 (22), 223503/1-223503/3. 